\title{
Suspicious myometrial mass on ultrasonography and MRI does not necessarily mean a sarcoma on histology
}

\author{
Jean-Christophe Lousse • Mathieu Jouret • \\ Etienne Marbaix • Latifa Fellah • Jean Squifflet • \\ Jacques Donnez
}

Received: 10 March 2009 / Accepted: 1 April 2009 /Published online: 29 April 2009

(C) Springer-Verlag 2009

\begin{abstract}
We report an unusual ultrasonographic and magnetic resonance imaging (MRI) presentation of a myometrial mass in a 38-year-old woman hoping to conceive. Hysterectomy had been proposed elsewhere because of the suspicious nature of the mass, but the patient was seeking a second opinion. This atypical formation looked consistent with either hydropic degeneration of a uterine myoma or leiomyosarcoma, but preoperative differential diagnosis was impossible. Laparoscopic tumorectomy was performed and histology confirmed a degenerating uterine myoma. We, therefore, show that unusual ultrasonographic and MRI findings do not necessarily require radical surgery, even if sarcoma cannot be excluded preoperatively, especially in patients who wish to conceive.
\end{abstract}

Keywords Uterine myoma - Leiomyosarcoma .

Ultrasonography $\cdot$ MRI $\cdot$ Laparoscopic myomectomy

\section{Case report}

A 38-year-old woman with an unusual myometrial mass presented to our department for a second opinion, having

J.-C. Lousse $\cdot$ M. Jouret $\cdot$ J. Squifflet $\cdot$ J. Donnez $(\bowtie)$

Department of Gynecology, Cliniques Universitaires Saint-Luc, Université Catholique de Louvain,

Avenue Hippocrate 10,

1200 Brussels, Belgium

e-mail: Jacques.Donnez@Uclouvain.be

\section{E. Marbaix}

Department of Pathology, Université Catholique de Louvain, 1200 Brussels, Belgium

L. Fellah

Department of Radiology, Université Catholique de Louvain, 1200 Brussels, Belgium been advised to have a hysterectomy in another institution. The patient was nulliparous but hoped to conceive in the future and had no symptoms. Clinical examination revealed a mobile uterus with a 6- to 7-week uterine volume.

Transvaginal ultrasonography showed an intramyometrial right-lateralized mass of $3 \mathrm{~cm}$, presenting as a heterogeneous, solid, and well-vascularized formation surrounded by a liquid halo (Fig. 1a). Pelvic magnetic resonance imaging (MRI) confirmed the presence of this atypical, well-circumscribed myometrial mass measuring $33 \times 20 \times 26 \mathrm{~mm}$, containing different components. Peripheral liquid was clearly visible with solid components of the mass enhanced on contrast images and a central nonenhanced area (Fig. 1b-d). This myometrial formation was undoubtedly unusual, consistent with hydropic degeneration of a myoma, although preoperative differential diagnosis with leiomyosarcoma was impossible. No lymph node invasion or peritoneal carcinosis was suspected on MRI.

Laparoscopy was, therefore, planned to assess the diagnosis. The peritoneal cavity was closely inspected and showed no signs of peritoneal carcinosis. The uterine serosa was opened, revealing a dense protruding mass with a cystic component inside the myometrium (Fig. 2a-c). A relatively welldelimited plane of cleavage was initially found, allowing dissection by traction and countertraction of the uterus. At greater depth, the mass was less demarcated and was entirely removed by sharp dissection (Fig. 2d). During resection, the macroscopic aspect of the formation was wholly consistent with a myoma. However, to avoid morcellation in the peritoneal cavity, a $\mathrm{LapSac}^{\circledR}$ was used to remove the mass.

Peritoneal cytology was negative and macroscopic section through the lesion showed a myomatous whitish nodule (Fig. 3a, b). Histology identified a cellular myoma (Fig. 3c) surrounded by areas of paucicellular hyaline fibrosis (Fig. 3d) [1]. Immunohistochemical labeling of phosphory- 
Fig. 1 Ultrasonographic and MRI imaging of the myometrial mass. Ultrasonography (a) showed an intramyometrial right-lateralized mass of $3 \mathrm{~cm}$, presenting as a heterogeneous, solid, and well-vascularized formation (solid arrow) surrounded by a liquid halo (dotted arrow). MRI sagittal (b) and coronal (c) and contrast-enhanced fat-suppressed T1-weighted (b) images showed a round, well-defined mass in the myometrium. This mass contained liquid (dotted arrow), as well as enhanced and nonenhanced solid components. Coronal views $(\mathbf{c}, \mathbf{d})$ showed a right ovarian cyst
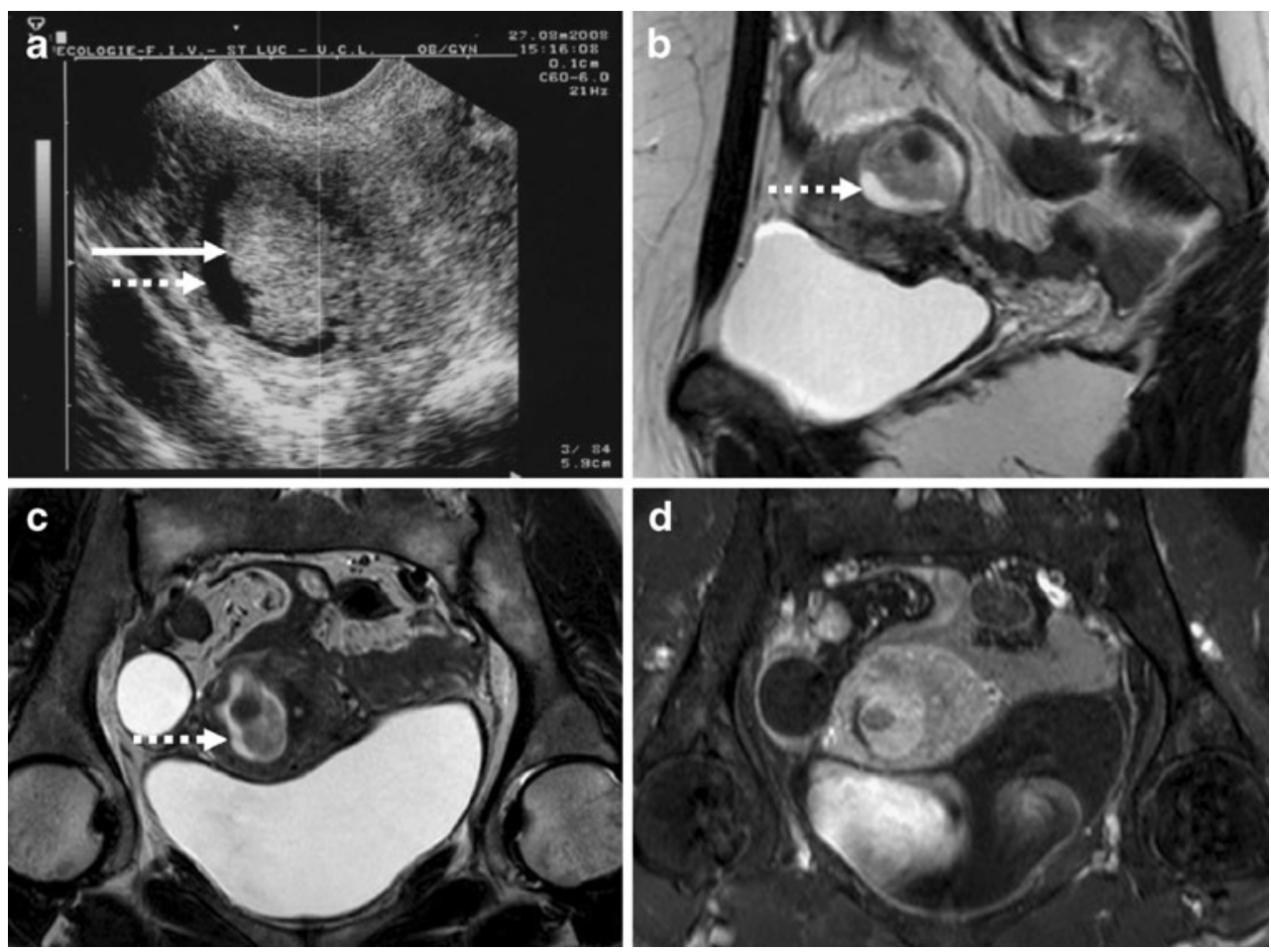
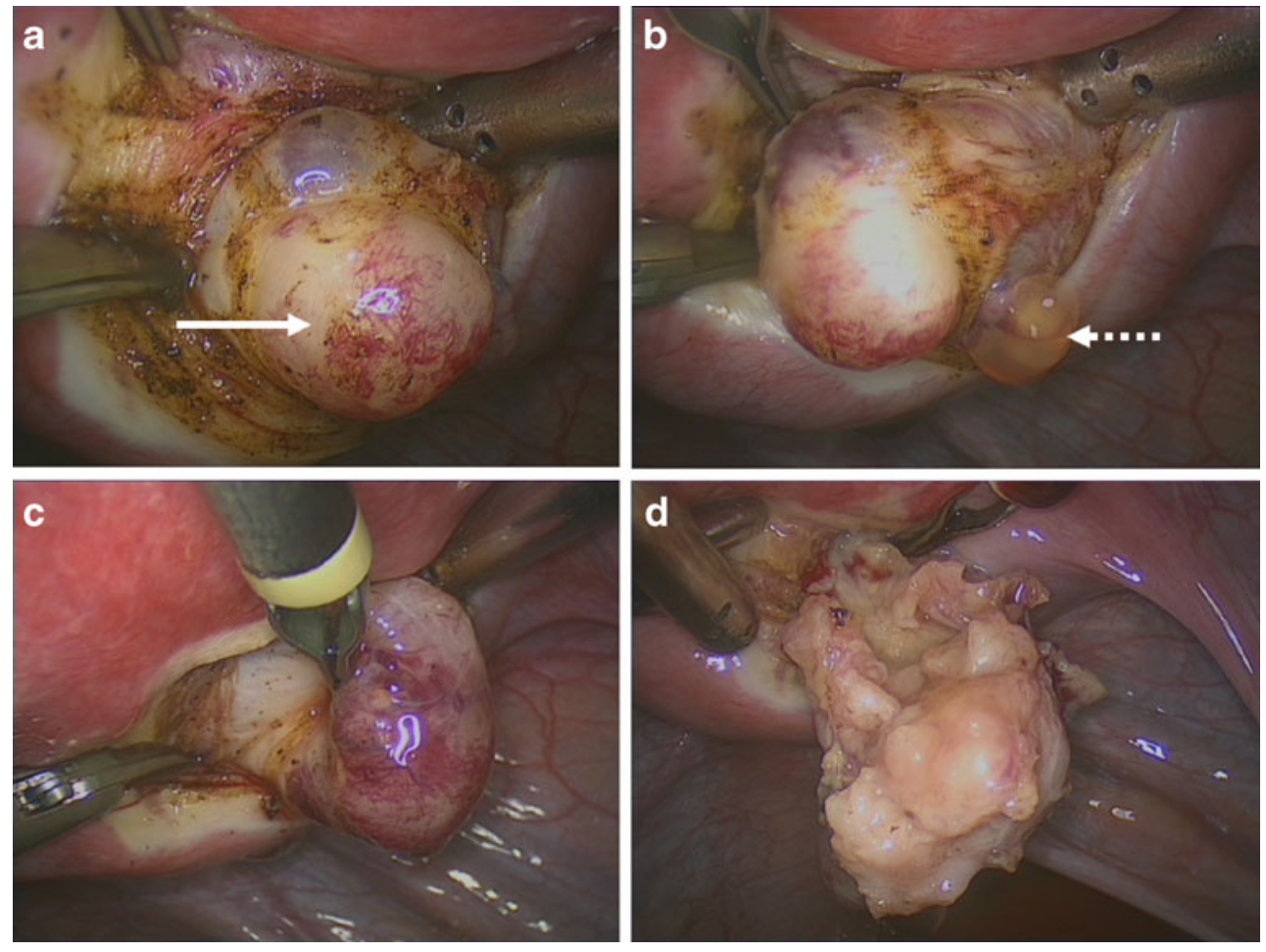

Fig. 2 Laparoscopic myomectomy. At laparoscopy, the uterine serosa was opened, revealing a dense protruding mass inside the myometrium (solid arrow) with a cystic component (dotted arrow) (Fig. 3a-c). A well-delimited plane of cleavage was initially found, allowing

dissection by traction and countertraction of the uterus. At greater depth, the mass was less demarcated and was entirely removed by coagulation and scissor excision (Fig. 3d) 
Fig. 3 Macroscopic and microscopic analyses of the removed myometrial mass. Macroscopic section through the lesion showed a whitish myomatous nodule $(\mathbf{a}, \mathbf{b}$; solid arrow). Histology confirmed the diagnosis of a cellular myoma (c) surrounded by areas of paucicellular hyaline fibrosis (d). Immunolabeling of phosphorylated histone $\mathrm{H} 3$ showed a very low mitotic rate $(\mathbf{e})$
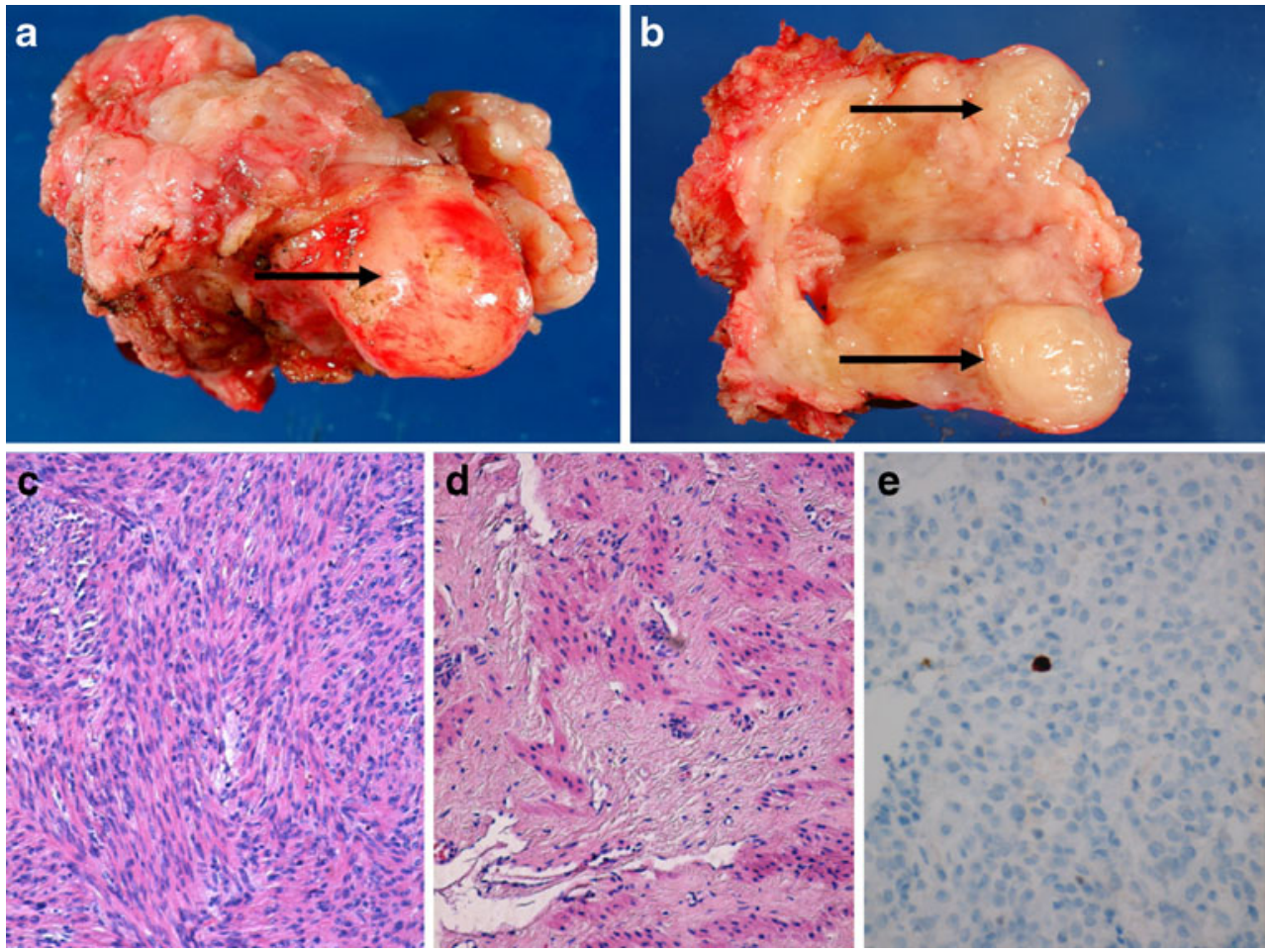

lated histone $\mathrm{H} 3$ showed a very low mitotic rate. The Ki67 proliferation index was less than $10 \%$ and immunohistochemical staining of desmin, alpha-actin, and caldesmon confirmed the muscular nature of the tumor.

\section{Conclusion}

In case of preoperative diagnosis of leiomyosarcoma, hysterectomy and bilateral salpingo-oophorectomy by laparotomy are the gold standard [2]. In our case, MRI presentation of a well-circumscribed mass was consistent with hydropic degeneration of a myoma, although leiomyosarcoma could not be excluded preoperatively. No lymph node invasion or peritoneal carcinosis was suspected on MRI.

Laparoscopy was, therefore, planned to assess our diagnosis. The peritoneal cavity was closely inspected to exclude peritoneal carcinosis. The uterine serosa was opened and a relatively well-delimited plane of cleavage was found. We believe that the risk of myometrial incision and associated peritoneal dissemination in case of sarcoma would have been similar by laparotomy or laparoscopy. During dissection, the macroscopic aspect of the formation was wholly consistent with a myoma. In case of suspected malignancy, extemporaneous histological analysis would have been required. Every precaution was taken to avoid rupturing the mass and a $\mathrm{LapSac}^{\circledR}$ was used to remove it in order to avoid morcellation in the peritoneal cavity.

Clinicians should be aware that unusual ultrasonographic and MRI findings do not necessarily require radical surgery, even if sarcoma cannot be excluded preoperatively, especially in patients who wish to conceive. Furthermore, as myomas are remarkably common, we believe that it is crucial to be familiar with unusual ultrasonographic and MRI presentations that may occur in case of degeneration.

Acknowledgements The authors thank Mira Hryniuk for reviewing the English grammar and syntax of the manuscript.

Disclosure of interests None.

\section{References}

1. Toledo G, Oliva E (2008) Smooth muscle tumors of the uterus. Arch Pathol Lab Med 132:595-605

2. Morice P, Rodrigues A, Pautier P, Rey A, Camatte S, Atallah D, Pomel C, Lhommé C, Haie-Meder C, Duvillard P, Castaigne D (2003) Surgical procedures for uterine sarcoma. Gynecol Obstet Fertil 31:147-150 